\title{
Reductions of partizan games
}

\author{
J.P. GROSSMAN AND AARON N. SIEGEL
}

\begin{abstract}
The reduced canonical form of a game $G$, denoted by $\bar{G}$, is the simplest game infinitesimally close to $G$. Reduced canonical forms were introduced by Calistrate [2], who gave a simple construction for computing $\bar{G}$. We provide a new correctness proof of Calistrate's algorithm, and show that his techniques generalize to produce a family of reduction operators. In addition, we introduce a completely new construction of $\bar{G}$, motivated by Conway's original canonical-form construction.
\end{abstract}

\section{Introduction}

Although canonical forms sometimes reveal substantial information about the structure of combinatorial games, they are often too complicated to be of any great use. Many of the most interesting games — including Clobber, Amazons, and Hare and Hounds - give rise to some massively complex canonical forms even on relatively small boards. In such cases, a method for extracting more specific information is highly desirable. The familiar temperature theory, and the theory of atomic weights for all-small games, can be viewed as efforts to address this problem.

In 1996, Dan Calistrate [2] introduced another type of reduction. Calistrate observed that in certain situations, infinitesimal differences are of secondary importance. He proposed associating to each game $G$ a reduced canonical form, $\bar{G}$, such that $\bar{G}=\bar{H}$ whenever $G-H$ is infinitesimal.

Calistrate's original construction defined $\bar{G}$ to be the simplest game equivalent to $G$ modulo an infinitesimal, where simplicity is measured in terms of the number of edges in the complete game tree. He gave a method for calculating $\bar{G}$ and claimed that the map $G \mapsto \bar{G}$ was a homomorphism. However, his proof of this assertion contained a flaw. 
Section 2 reviews Calistrate's construction, introduces some important definitions and notation, and establishes some basic results regarding infinitesimals. Section 3 introduces the group of even-tempered games. In Section 4, we give a natural definition of reduced canonical form, and show that it matches Calistrate's construction. In Section 5, we show that many of our results generalize to a broad family of homomorphisms of the group of games. Section 6 establishes the relationship between these homomorphisms and reduced canonical forms. Finally, Section 7 poses some interesting open problems.

\section{Preliminaries}

Throughout this paper we use the equivalent terms form and representation to denote a particular formal representation of a game $G$. Given a game $G=\left\{G^{L}\right.$ | $G^{R}$, we will use the terms followers to mean all subpositions of $G$, including $G$ itself; proper followers to mean all subpositions of $G$, excluding $G$; and options to mean the immediate subpositions $G^{L}, G^{R}$.

A game $\varepsilon$ is an infinitesimal if, for every positive number $x$, we have $-x<$ $\varepsilon<x$. Let Inf denote the set of infinitesimals; clearly Inf is a subgroup of $\mathcal{G}$, the group of games. When $G-H$ is infinitesimal, we say that $G$ and $H$ are infinitesimally close, and write $G \equiv \equiv_{\operatorname{Inf}} H$. We will sometimes say that $H$ is $G$-ish ( $G$ infinitesimally shifted).

DEFINITION 2.1. If $G$ is a game (in any form) and $\varepsilon \bowtie 0$ is an infinitesimal, then $G$ reduced by $\varepsilon$, denoted by $G_{\varepsilon}$, is defined by

$$
G_{\varepsilon}= \begin{cases}G & \text { if } G \text { is a number; } \\ \left\{G_{\varepsilon}^{L}-\varepsilon \mid G_{\varepsilon}^{R}+\varepsilon\right\} & \text { otherwise. }\end{cases}
$$

When no restriction is placed on the game $\varepsilon$, this operation is commonly known as unheating. It is not immediately evident that $G_{\varepsilon}=H_{\varepsilon}$ whenever $G=H$, but this will emerge in Section 5. In fact, we will prove the stronger statement that $G_{\varepsilon}=H_{\varepsilon}$ if and only if $G$ and $H$ are infinitesimally close. The special case $\varepsilon=*$ was first considered by Calistrate, who defined an additional operator to effect a further reduction:

Definition 2.2 (CAlistrate). If $G$ is a game (in any form), then the *projection of $G$, denoted by $p(G)$, is defined by

$$
p(G)= \begin{cases}x & \text { if } G=x \text { or } x+* \text { for some number } x, \\ \left\{p\left(G^{L}\right) \mid p\left(G^{R}\right)\right\} & \text { otherwise. }\end{cases}
$$

We can then define $\bar{G}=p\left(G_{*}\right)$. Calistrate claimed that $\bar{G}$ is the simplest game infinitesimally close to $G$. While this statement is correct, Calistrate's proof 
relied on the assertion that $G \mapsto \bar{G}$ is a homomorphism, which is false. For example, let $G=\{1 \mid 0\}$ and $H=\{2|| 1 \mid 0\}$. Then $G=\bar{G}$ and $H=\bar{H}$, so

$$
\bar{G}+\bar{H}=G+H=\{2,\{3 \mid 2\} \| 1\} \text {, but } \overline{G+H}=\{3 \mid 2 \| 1\} .
$$

We will give an alternate proof that $\bar{G}$ is the simplest game infinitesimally close to $G$. We shall have occasion to consider other mappings that select representatives of each Inf-equivalence class. Proceeding in maximum generality:

DEFINITION 2.3. A mapping $\rho: \mathcal{G} \rightarrow \mathcal{G}$ is a reduction (modulo Inf) if

(i) for all $G, \rho(G) \equiv_{\operatorname{Inf}} G$;

(ii) if $x$ is a number, then $\rho(x)=x$; and

(iii) for all $G, H$ with $G \equiv \operatorname{Inf} H$, we have $\rho(G)=\rho(H)$.

If $\rho$ is a reduction, then we say that $\rho(G)$ is the reduced form of $G$ (under $\rho$ ).

Note that we do not require our reductions to be homomorphisms. The definition on its own is not terribly restrictive, but serves as a useful checklist for verifying candidate mappings with other desirable properties. We will show that $G \mapsto \bar{G}$ is a reduction, and also that $G \mapsto G_{\varepsilon}$ is both a reduction and a homomorphism for any infinitesimal $\varepsilon \bowtie 0$.

Infinitesimals and stops. We will make extensive use of an equivalent definition of infinitesimal that is given in terms of the stops of a game. Recall, from Winning Ways, that the Left (Right) stop of $G$ is equal to the first number reached when $G$ is played optimally in isolation, with Left (Right) moving first. Formally:

DEFINITION 2.4. The Left and Right stops of $G$, denoted by $L_{0}(G)$ and $R_{0}(G)$, are defined recursively by

$$
\begin{aligned}
& L_{0}(G)= \begin{cases}G & \text { if } G \text { is a number } \\
\max R_{0}\left(G^{L}\right) & \text { otherwise }\end{cases} \\
& R_{0}(G)= \begin{cases}G & \text { if } G \text { is a number } \\
\min L_{0}\left(G^{R}\right) & \text { otherwise }\end{cases}
\end{aligned}
$$

The following facts about stops, and their relationship to infinitesimals, will be used throughout the rest of this paper. Some proofs can be found in [3]; the rest are simple exercises left to the reader.

Proposition 2.5. Let $G, H$ be any games.

(a) $G$ is an infinitesimal if and only if $L_{0}(G)=R_{0}(G)=0$.

(b) $R_{0}(G)+R_{0}(H) \leq R_{0}(G+H) \leq R_{0}(G)+L_{0}(H)$;

$$
L_{0}(G)+L_{0}(H) \geq L_{0}(G+H) \geq R_{0}(G)+L_{0}(H) \text {. }
$$


(c) If $G \geq H$, then $L_{0}(G) \geq L_{0}(H)$ and $R_{0}(G) \geq R_{0}(H)$.

(d) $L_{0}(G) \geq R_{0}\left(G^{L}\right)$ and $R_{0}(G) \leq L_{0}\left(G^{R}\right)$ for all $G^{L}$, $G^{R}$, even when $G$ is a number.

Infinitesimal comparisons. We can define infinitesimal comparisons in a manner similar to our definition of infinitesimally close:

DEFINITION 2.6. $G \geq_{\text {Inf }} H$ if and only if $G \geq H+\varepsilon$ for some infinitesimal $\varepsilon$; $G \leq_{\text {Inf }} H$ is defined similarly.

Note that $G \equiv_{\operatorname{Inf}} H$ if and only if $G \leq_{\operatorname{Inf}} H$ and $G \geq_{\operatorname{Inf}} H$. We will see shortly that in these definitions it suffices to take $\varepsilon$ to be some multiple of $\uparrow$ or $\downarrow$. We begin with:

LEMMA 2.7. If $R_{0}(G) \geq 0$, then $G \geq n \cdot \downarrow$ for some $n$.

Proof. Choose $n>$ birthday $(G)+1$. Then Left, playing second, can win $G+n \cdot \uparrow$ as follows: He makes all of his moves in $G$, playing optimally, until that component reaches a number $x$. Then, since $L_{0}(H) \geq R_{0}(H)$ for all followers $H$ of $G$, we must have $x \geq 0$. By the assumptions on $n$, Left still has a move to 0 available in the $n \cdot \uparrow$ component (even if all of Right's moves were in that component), so he wins on his next move.

By symmetry, if $L_{0}(G) \leq 0$, then $G \leq n \cdot \uparrow$ for some $n$.

COROllary 2.8. Let $G$ and $H$ be games.

(a) $G \geq_{\operatorname{Inf}} H$ if and only if $G-H \geq n \cdot \downarrow$ for some $n$.

(b) $G$ is infinitesimal if and only if $n \cdot \downarrow \leq G \leq n \cdot \uparrow$ for some $n$.

PROOF. Follows immediately from Lemma 2.7 and Proposition 2.5(a).

Lemma 2.7 also allows us to restate a well-known incentive theorem in terms of infinitesimal comparisons:

THEOREM 2.9. If $G$ is not a number, then $G$ has at least one Left incentive and at least one Right incentive that are $\geq_{\operatorname{Inf}} 0$.

Proof. Let $G^{L}$ be any Left option with $R_{0}\left(G^{L}\right)=L_{0}(G)$. Then

$$
R_{0}\left(G^{L}-G\right) \geq R_{0}\left(G^{L}\right)+R_{0}(-G)=L_{0}(G)-L_{0}(G)=0 .
$$

Hence $G^{L}-G \geq_{\text {Inf }} 0$ by Lemma 2.7. The proof for Right incentives is identical.

We conclude with a theorem that is intuitive yet difficult to prove without the preceding machinery; the theorem states that if the options of a game are infinitesimally perturbed, the resulting game is infinitesimally close to the original. 
THEOREM 2.10. If $G=\left\{G^{L} \mid G^{R}\right\}$ is not a number and $G^{\prime}=\left\{G^{L^{\prime}} \mid G^{R^{\prime}}\right\}$ is a game with $G^{L^{\prime}} \equiv_{\mathrm{Inf}} G^{L}$ and $G^{R^{\prime}} \equiv_{\operatorname{Inf}} G^{R}$, then $G^{\prime} \equiv_{\operatorname{Inf}} G$.

PROOF. By symmetry it suffices to show that $G-G^{\prime} \geq_{\text {Inf }} 0$, or equivalently (Corollary 2.8) that $G-G^{\prime}+n \cdot \uparrow \geq 0$ for sufficiently large $n$. If Right moves in $G$ or $-G^{\prime}$, Left answers with the corresponding move in $-G^{\prime}$ or $G$ and wins with $n$ large enough by Corollary 2.8. If Right moves to $G-G^{\prime}+(n-1) \cdot \uparrow *$, then by Theorem 2.9 there is some $G^{R} \leq_{\operatorname{Inf}} G$, so Left moves in $-G^{\prime}$ to $-G^{R^{\prime}} \equiv \equiv_{\operatorname{Inf}}-G^{R}$. Again, Left wins with $n$ large enough by Corollary 2.8 since $G-G^{R^{\prime}} \geq \operatorname{Inf} 0$.

Note that Theorem 2.10 fails if $G$ is a number. For example, $0 \equiv_{\operatorname{Inf}} *$, but $\{0 \mid 1\}=\frac{1}{2}$ and $\{* \mid 1\}=0$.

\section{Temper}

By Proposition 2.5, infinitesimal differences do not change the final score of a game; they affect only who has the move when that score is reached. This observation motivates one of the most natural reduced-form constructions. Loosely speaking, call a game $G$ even-tempered if, no matter how $G$ is played, the first player will have the move when $G$ reaches a number. If $G$ and $H$ are infinitesimally close and even-tempered then we should expect that $G=H$, since we have effectively discarded the particularities of who has the move and when. This is indeed the case, and in fact we can prove a stronger statement: For any game $G, G_{*}$ is the unique even-tempered game infinitesimally close to $G$. We begin with a formal definition of temper.

DEFINITION 3.1. Let $G$ be a fixed representation of a game.

(a) $G$ is even-tempered if $G$ a number, or every option of $G$ is odd-tempered;

(b) $G$ is odd-tempered if $G$ is not a number and every option of $G$ is eventempered;

(c) $G$ is well-tempered if $G$ is even-tempered or odd-tempered.

We will call a game even- (odd-, well-) tempered if it has some even- (odd-, well-) tempered representation. Although temper is a property of the form of a game and can be destroyed by adding new dominated options, the following provides justification for treating it as a property of games:

THEOREM 3.2. Let $G$ be a game in any form. If $G$ is even- (odd-) tempered, then so is its canonical form.

PROOF. If $G$ is a number then both $G$ and its canonical form are necessarily even-tempered, so assume that it is not. By induction we may assume that all proper followers of $G$ are canonical. It then suffices to show that temper is 
preserved when dominated options are eliminated or reversible moves bypassed. For dominated options this is trivial, so suppose some $G^{L R} \leq G$.

Consider the case where $G^{L R}$ is a number. Then $G \neq G^{L R}$, so by the Number Avoidance Theorem, Left has a winning move from $G-G^{L R}$ to some $G^{L^{\prime}}$ $G^{L R}$. Thus $G^{L^{\prime}} \geq G^{L R}$. But since $G^{L R}$ is a number, we have $G^{L R}>G^{L R L}$, so any $G^{L R L}$ is dominated by $G^{L^{\prime}}$ and hence does not contribute to the canonical form of $G$.

If $G^{L R}$ is not a number then neither is $G^{L}$ (since it was assumed canonical), and since $G^{L}$ is odd- (even-) tempered, so is every $G^{L R L}$.

A simple corollary is that a game cannot be both odd- and even- tempered since its canonical form cannot be both. The main theorem of this section is the following:

THEOREM 3.3. Let $G$ be a game in any form. Then $G_{*}$ is the unique $G$-ish even-tempered game.

Theorem 3.3 implies that $G_{*}=H_{*}$ whenever $G=H$, which is not immediately clear from the definition of $G_{*}$. For now, we must specify a representation for $G$ in order to compute $G_{*}$. Several lemmas are critical to the proof of Theorem 3.3.

LeMma 3.4. Let $G, H$ be any games.

(a) If $G$ and $H$ are both even- (odd-) tempered, then $G+H$ is even-tempered.

(b) If $G$ is even-tempered and $H$ is odd-tempered, then $G+H$ is odd-tempered.

Proof. If $G$ and $H$ are both numbers then so is $G+H$, and the conclusion follows. If $G$ is a number and $H$ is not, then by the Number Avoidance Theorem

$$
G+H=\left\{G+H^{L} \mid G+H^{R}\right\} .
$$

By induction $G+H^{L}$ and $G+H^{R}$ have the same temper as $H^{L}$ and $H^{R}$. Finally, if neither $G$ nor $H$ is a number, then

$$
G+H=\left\{G^{L}+H, G+H^{L} \mid G^{R}+H, G+H^{R}\right\} .
$$

By induction and assumption on $G, H$ all options have the same temper. Furthermore, they are odd-tempered if $G$ and $H$ have the same temper, and eventempered otherwise.

LEMMA 3.5. Let $G$ be a game in any form. Then $G_{*}$ is even-tempered and infinitesimally close to $G$.

Proof. The conclusion is trivial if $G$ is a number. If $G$ is not a number then $G_{*}=\left\{G_{*}^{L}+* \mid G_{*}^{R}+*\right\}$, and the result follows from induction, Lemma 3.4, Theorem 2.10, and the fact that $*$ is odd-tempered. 
LEMMA 3.6. If $G$ is even-tempered and $R_{0}(G) \geq 0$, then $G \geq 0$.

ProOF. We may assume that $G$ is in canonical form. Since $R_{0}(G) \geq 0$, Left, playing second, can assure that when the play reaches a number, the result is $\geq 0$. By Theorem 3.2, this necessarily happens after an even number of moves, so Left has made the last move. Thus, Left can win $G$ as second player.

Proof of Theorem 3.3. Lemma 3.5 shows that $G_{*}$ is even-tempered. For uniqueness, suppose $G$ and $H$ are infinitesimally close even-tempered games. Then $R_{0}(G-H)=0$. But $G-H$ is even-tempered, so by Lemma 3.6, we have $G-H \geq 0$; by symmetry $G-H=0$.

As a simple corollary, $G_{*}+*$ is the unique $o d d$-tempered game infinitesimally close to $G$. A more substantial corollary is the following theorem.

THEOREM 3.7. The map $G \mapsto G_{*}$ is a well-defined homomorphism of the group of games.

PROOF. First, Theorem 3.3 shows that $G_{*}$ does not depend on the form of $G$. Now fix games $G, H$. Lemma 3.5 implies that

$$
(G+H)_{*} \equiv_{\operatorname{Inf}} G+H \equiv_{\operatorname{Inf}} G_{*}+H_{*} .
$$

Also, from Lemmas 3.4 and 3.5, we know that $(G+H)_{*}$ and $G_{*}+H_{*}$ are both even-tempered. It follows from Theorem 3.3 that they are equal.

As a final note, Lemma 3.4 shows that the well-tempered games $\mathcal{W}$ and the even-tempered games $\mathcal{E}$ are subgroups of the group of games. Moreover, the mapping $G \mapsto G+*$ induces a perfect pairing of even- and odd- tempered games, so that

$$
\mathcal{W}=\mathcal{E} \cup\{G+*: G \in \mathcal{E}\} .
$$

Thus, the index of $\mathcal{E}$ in $\mathcal{W}$ is 2 .

\section{Reduced canonical forms}

In this section we will show that every game $G$ has a reduced canonical form $\bar{G} . \bar{G}$ is infinitesimally close to $G$, and it is the simplest such game, in a sense that we will define shortly.

DEFINITION 4.1. Let $G$ be any game.

(a) A Left option $G^{L}$ is Inf-dominated if $G^{L} \leq_{\operatorname{Inf}} G^{L^{\prime}}$ for some other Left option $G^{L^{\prime}}$.

(b) A Left option $G^{L}$ is Inf-reversible if $G^{L R} \leq_{\operatorname{Inf}} G$ for some $G^{L R}$.

The definitions for Right options are similar. 
ExAMPLE (i). Let $G=\{1,\{2 \mid 1\} \| 0\}$. Then 1 is an Inf-dominated Left option of $G$, since $\{2 \mid 1\}-1+\uparrow \geq 0$.

EXAmple (ii). Let $H=\{1,\{2 \mid 0\} \| 0\}$. Then $\{2 \mid 0\}$ is Inf-reversible through 0 , since $H-0+\uparrow \geq 0$.

DEFINITION 4.2. A game $G$ is said to be in reduced canonical form if, for every follower $H$ of $G$, either

(i) $H$ is a number in simplest form, or

(ii) $H$ is not numberish, and contains no Inf-dominated or Inf-reversible options.

This definition of reduced canonical form appears radically different from Calistrate's, but we will soon see that his construction meets our criteria. The main theorems exactly parallel the corresponding results for canonical forms [3]:

THEOREM 4.3. For any game $G$, there is a game $\bar{G}$ in reduced canonical form with $\bar{G} \equiv{ }_{\text {Inf }} G$.

THEOREM 4.4. Suppose that $G$ and $H$ are in reduced canonical form. If $G \equiv_{\operatorname{Inf}}$ $H$, then $G=H$.

Theorem 4.4 guarantees that the $\bar{G}$ found in Theorem 4.3 is unique. The following lemma is instrumental to the proof of Theorem 4.3.

LEMMA 4.5. Let $G$ be a well-tempered game.

(a) If $G \equiv_{\operatorname{Inf}} x$ for some number $x$, then $G=x$ or $x+*$.

(b) If $G$ is in canonical form, then $G$ has no Inf-dominated or Inf-reversible options.

(c) $p(G) \equiv_{\text {Inf }} G$.

ProOf. (a) $x$ is the unique even-tempered $x$-ish game, so if $G$ is even-tempered then $G=x$. Likewise, $x+*$ is the unique odd-tempered $x$-ish game, so if $G$ is odd-tempered then $G=x+*$.

(b) First suppose (for contradiction) that $G^{L} \leq_{\text {Inf }} G^{L^{\prime}}$ for distinct Left options $G^{L}, G^{L^{\prime}}$. Since $G$ is even- (odd-) tempered, both $G^{L}$ and $G^{L^{\prime}}$ are odd- (even-) tempered. Therefore $G^{L^{\prime}}-G^{L}$ is even-tempered. But since $G^{L} \leq_{\text {Inf }} G^{L^{\prime}}$, we know that $R_{0}\left(G^{L^{\prime}}-G^{L}\right) \geq 0$. By Lemma 3.6, this implies that $G^{L^{\prime}}-G^{L} \geq 0$, contradicting the assumption that $G$ is in canonical form.

Next suppose (for contradiction) that $G^{L R} \leq_{\text {Inf }} G$. Consider the case where $G^{L}$ is a number. Then $G^{L R}$ is also a number and $G^{L}<G^{L R}$. Furthermore, by Theorem 2.9, there is some $G^{L^{\prime}} \geq \operatorname{Inf} G$. Hence

$$
G^{L}<G^{L R} \leq_{\operatorname{Inf}} G \leq_{\operatorname{Inf}} G^{L^{\prime}} .
$$


Since $G^{L R}$ and $G^{L}$ necessarily differ by more than an infinitesimal, this gives us $G^{L}<G^{L^{\prime}}$, contradicting the assumption that $G$ is in canonical form.

Finally, if $G^{L}$ is not a number, then $G^{L R}$ is well-tempered and has the same temper as $G$. So $G-G^{L R}$ is even-tempered. But $R_{0}\left(G-G^{L R}\right) \geq 0$, so by Lemma 3.6 we have $G-G^{L R} \geq 0$, again contradicting the assumption that $G$ is in canonical form.

(c) follows immediately from induction and Theorem 2.10.

Proof of Theorem 4.3. We will show that $p\left(G_{*}\right)$ has the desired properties, where we use the canonical form of $G_{*}$ to compute $p\left(G_{*}\right)$. Let $H$ be a follower of $p\left(G_{*}\right)$; then $H=p\left(H^{\prime}\right)$ for some follower $H^{\prime}$ of $G_{*}$. Since $G_{*}$ is welltempered and in canonical form, the same is true of $H^{\prime}$.

If $H$ is numberish, then by Lemma 4.5(c), so is $H^{\prime}$. By Lemma 4.5(a), we have $H^{\prime}=x$ or $x+*$ for some number $x$; then by definition, $H=x$. This verifies condition (i) in the definition of reduced canonical form.

If $H$ is not numberish, then by Lemma 4.5(c), neither is $H^{\prime}$. By Lemma 4.5(b), $H^{\prime}$ has no Inf-dominated or Inf-reversible options. Since all followers of $H$ are infinitesimally close to followers of $H^{\prime}$, the same must be true of $H$. This completes the proof.

We are now ready to prove uniqueness (Theorem 4.4).

Proof of Theorem 4.4. Suppose $G$ and $H$ are in reduced canonical form and $G \equiv_{\operatorname{Inf}} H$. If either of $G, H$ is numberish then both must be, so by the definition of reduced canonical form, both are numbers; hence $G=H$.

Now suppose that neither $G$ nor $H$ is numberish, and consider $H-G$. Since $R_{0}(H-G) \geq 0$, by Corollary 2.8 we have

$$
H-G+n \cdot \uparrow \geq 0
$$

for suitably large $n$. Consider the game after Right moves to $-G^{L}$ :

$$
H-G^{L}+n \cdot \uparrow
$$

Left must have a winning response. It cannot be to any $H-G^{L R}+n \cdot \uparrow$, since this would imply

$$
G \equiv_{\operatorname{Inf}} H \geq_{\text {Inf }} G^{L R},
$$

contradicting the assumption that $G$ has no Inf-reversible options. Furthermore, by Theorem 2.9, $H$ has a Left incentive that exceeds $n \cdot \downarrow$ (assuming $n$ is sufficiently large). Since $n \cdot \downarrow$ is the unique Left incentive of $n \cdot \uparrow$, Left must have a winning move in $H$, to

$$
H^{L}-G^{L}+n \cdot \uparrow
$$


Therefore $H^{L} \geq_{\operatorname{Inf}} G^{L}$. By an identical argument, there is some $G^{L^{\prime}}$ with $G^{L^{\prime}} \geq$ Inf $H^{L}$. But $G$ has no Inf-dominated options, so in fact

$$
G^{L^{\prime}} \equiv_{\operatorname{Inf}} H^{L} \equiv_{\operatorname{Inf}} G^{L} .
$$

By induction, $G^{L}=H^{L}$, so every Left option of $G$ is a Left option of $H$. Symmetrical arguments show that $G$ and $H$ have exactly the same Left and Right options.

If $G$ is any game, the value of $G$ is unchanged when dominated options are eliminated or reversible ones bypassed. We can similarly eliminate Inf-dominated options and bypass Inf-reversible ones, preserving the value of $G$ up to an infinitesimal.

LEMMA 4.6. If $G$ is not a number and $G^{\prime}$ is obtained from $G$ by eliminating an Inf-dominated option, then $G^{\prime} \equiv_{\operatorname{Inf}} G$.

Proof. Suppose that $G^{L^{\prime}} \leq$ Inf $G^{L}$ for Left options $G^{L}, G^{L^{\prime}}$ of $G$. Then we can find $H \equiv_{\operatorname{Inf}} G^{L}$ such that $G^{L^{\prime}} \geq H$, so, by Theorem 2.10 ,

$$
G=\left\{G^{L}, G^{L^{\prime}}, \ldots \mid G^{R}\right\} \equiv_{\operatorname{Inf}}\left\{G^{L^{\prime}}, H, \ldots \mid G^{R}\right\}=\left\{G^{L^{\prime}}, \ldots \mid G^{R}\right\} .
$$

LEMMA 4.7. If $G$ is not numberish and $G^{\prime}$ is obtained from $G$ by bypassing an Inf-reversible option, then $G^{\prime} \equiv_{\mathrm{Inf}} G$.

Note that the assumption of Lemma 4.7 ( $G$ not numberish) is stronger than the assumption of Lemma 4.6 ( $G$ not a number). If $G$ is numberish, Inf-reversible moves cannot in general be bypassed. For example, let $G=-2=\{2 \mid 0 \| 0\}$. Then $G^{L R}=0 \leq_{\text {Inf }} G$, but if we replace $G^{L}=\{2 \mid 0\}$ with the Left options of 0 , then the resulting game is $G^{\prime}=\{\mid 0\}=-1 \not_{\text {Inf }} G$.

Proof of Lemma 4.7. Suppose that $G$ is not numberish, and $G^{\prime}$ is obtained from $G$ by bypassing some Inf-reversible option $G^{L_{0}}$ through $G^{L_{0} R_{0}}$. We must show that $G-G^{\prime}+n \cdot \uparrow \geq 0$ and $G^{\prime}-G+n \cdot \uparrow \geq 0$ for sufficiently large $n$.

First consider $G-G^{\prime}+n \cdot \uparrow \geq 0$. Right has three possible opening moves; we show that, in each case, Left has a winning response.

(a) If Right moves to $G-G^{\prime}+(n-1) \cdot \uparrow *$, then by Theorem 2.9 Left can move to $G-G^{R}+(n-1) \cdot \uparrow *$ with $G^{R} \leq_{\text {Inf }} G$ which wins for large enough $n$.

(b) Suppose Right moves to $G-G^{L_{0} R_{0} L}+n \cdot \uparrow$. Since $G^{L_{0} R_{0}} \leq_{\text {Inf }} G$, we have $G-G^{L_{0} R_{0}}+n \cdot \uparrow \geq 0$ for large enough $n$, so $G-G^{L_{0} R_{0} L}+n \cdot \uparrow \bowtie 0$ and therefore Left has a winning move.

(c) If Right makes any other move in $G$ or $-G^{\prime}$, Left makes the corresponding move in the other component leaving $n \cdot \uparrow$.

Now consider $G-G^{\prime}+n \cdot \uparrow \geq 0$. Once again, there are three cases. 
(d) Suppose that Right moves to $G^{\prime}-G+(n-1) \cdot \uparrow *$. Since $G$ is not a number, by Theorem 2.9 we can choose some $G^{L} \geq$ Inf $G$. If we can choose $G^{L} \neq G^{L_{0}}$, Left moves to $G^{L}-G+(n-1) \cdot \uparrow *$ and wins for large $n$. If $G^{L_{0}}$ is the only choice, the proof of Theorem 2.9 shows that $R_{0}\left(G^{L_{0}}\right)=L_{0}(G)$. We also have from Proposition 2.5 that $R_{0}\left(G^{L_{0} R_{0}}\right) \leq R_{0}(G)$ since $G^{L_{0} R_{0}} \leq_{\text {Inf }} G$. Finally, since $G$ is not numberish, we have $R_{0}(G)<L_{0}(G)$. Putting these inequalities together gives us

$$
R_{0}\left(G^{L_{0} R_{0}}\right) \leq R_{0}(G)<L_{0}(G)=R_{0}\left(G^{L_{0}}\right) \leq L_{0}\left(G^{L_{0} R_{0}}\right)
$$

and therefore $G^{L_{0} R_{0}}$ is not a number. It follows that $L_{0}\left(G^{L_{0} R_{0}}\right) \leq L_{0}\left(G^{\prime}\right)$ since every Left option of $G^{L_{0} R_{0}}$ is also a Left option of $G^{\prime}$ (by Proposition $2.5(\mathrm{~d})$ this is true even if $G^{\prime}$ is a number). Similarly, $R_{0}\left(G^{\prime}\right) \leq R_{0}(G)$, since they have the same Right options and $G$ is not a number. Hence

$$
R_{0}\left(G^{\prime}\right) \leq R_{0}(G)<L_{0}(G) \leq L_{0}\left(G^{L_{0} R_{0}}\right) \leq L_{0}\left(G^{\prime}\right)
$$

so in fact $G^{\prime}$ is also not a number. It follows from Theorem 2.9 that Left can win by moving to $G^{\prime}-G^{R}+(n-1) \cdot \uparrow *$, where $G^{R} \leq_{\operatorname{Inf}} G^{\prime}$.

(e) If Right moves to $G^{\prime}-G^{L_{0}}+n \cdot \uparrow$ then Left moves to $G^{\prime}-G^{L_{0} R_{0}}+n \cdot \uparrow$, which we now show is a loss for Right.

If Right moves to either $G^{R}-G^{L_{0} R_{0}}+n \cdot \uparrow$ or $G^{\prime}-G^{L_{0} R_{0}}+(n-1) \cdot \uparrow *$, Left can win with $n$ large enough since $-G^{L_{0} R_{0}} \geq_{\text {Inf }}-G$ and we have already shown that $G^{R}-G+n \cdot \uparrow \bowtie 0$ and $G^{\prime}-G+(n-1) \cdot \uparrow * \triangleright 0$. If Right moves to $G^{\prime}-G^{L_{0} R_{0} L}+n \cdot \uparrow$, Left makes the corresponding move in $G^{\prime}$ leaving $n \cdot \uparrow$ and wins.

(f) Finally, if Right makes any other move in $G^{\prime}$ or $-G$, Left makes the corresponding move in the other component leaving $n \cdot \uparrow$.

Lemmas 4.6 and 4.7 suggest an algorithm for computing $\bar{G}$. If $G$ is numberish, we take $\bar{G}=L_{0}(G)$. Otherwise, by Theorem 2.10 , we may assume that every option of $G$ is in reduced canonical form. $\bar{G}$ can then be obtained by iteratively eliminating Inf-dominated options and bypassing Inf-reversible ones until none remain.

This algorithm bears a pleasing similarity to the classical procedure for computing the canonical form of $G$, but it is somewhat less efficient than simply calculating $p\left(G_{*}\right)$. Nonetheless, Theorem 2.10 and Lemmas 4.6, 4.7 can be useful in practice; see, for example, Mesdal [4, Section 7] in this volume.

THEOREM 4.8. If $G$ is not numberish, then $\bar{G}$ can be computed by repeating the following steps in any order:

(i) Replacing options with simpler options infinitesimally close to the original;

(ii) Eliminating Inf-dominated options; 
(iii) Bypassing Inf-reversible options.

Proof. Since each step simplifies the game, the steps must at some point come to an end. Theorem 2.10 shows that step (i) does not change the game by more than an infinitesimal, and Lemmas 4.6 and 4.7 show that neither do steps (ii) or (iii). Thus, the final game is infinitesimally close to the original, and if no more steps are possible then all numberish followers are numbers and there are no Inf-dominated or Inf-reversible moves. It follows by Theorem 4.4 that this game is $\bar{G}$.

\section{Reduction by $\varepsilon$}

Having observed one reduction that is also a homomorphism, it is natural to look for others. In this section we show that for any infinitesimal $\varepsilon \Vdash \nabla$, the mapping $G \mapsto G_{\varepsilon}$ is both a reduction and a homomorphism. In the process we will show that $G_{\varepsilon}$ is well-defined, but for now we must still agree upon a specific representation of $G$ in order to compute $G_{\varepsilon}$.

LeMMA 5.1. $L_{0}\left(G_{\varepsilon}\right)=L_{0}(G)$ and $R_{0}\left(G_{\varepsilon}\right)=R_{0}(G)$.

Proof. This is immediate if $G$ is a number. Otherwise, by induction and Proposition 2.5, we have

$$
L_{0}\left(G_{\varepsilon}\right)=\max \left\{R_{0}\left(G_{\varepsilon}^{L}-\varepsilon\right)\right\}=\max \left\{R_{0}\left(G_{\varepsilon}^{L}\right)\right\}=\max \left\{R_{0}\left(G^{L}\right)\right\}=L_{0}(G),
$$

and similarly for $R_{0}$.

LEMMA 5.2. If $R_{0}(G) \geq 0$, then $G_{\varepsilon} \geq 0$; if $L_{0}(G) \leq 0$, then $G_{\varepsilon} \leq 0$.

Proof. Suppose $R_{0}(G) \geq 0$. If $G_{\varepsilon}$ is a number, then $G_{\varepsilon}=R_{0}\left(G_{\varepsilon}\right)=R_{0}(G) \geq$ 0 . Otherwise, suppose that Right moves in $G_{\varepsilon}$ to $G_{\varepsilon}^{R}+\varepsilon$. If $G_{\varepsilon}^{R}$ is a number, then

$$
G_{\varepsilon}^{R}=L_{0}\left(G_{\varepsilon}^{R}\right)=L_{0}\left(G^{R}\right) \geq R_{0}(G) \geq 0,
$$

so by choice of $\varepsilon, G_{\varepsilon}^{R}+\varepsilon \gg 0$ and Left has a winning move. Finally, if $G_{\varepsilon}^{R}$ is not a number, Left moves to $G_{\varepsilon}^{R L}$, choosing $G^{R L}$ so that $R_{0}\left(G^{R L}\right)=L_{0}\left(G^{R}\right)$. Since $L_{0}\left(G^{R}\right) \geq R_{0}(G) \geq 0$, by induction $G_{\varepsilon}^{R L} \geq 0$, so Left wins. The proof for $L_{0}(G)$ is identical.

THEOREM 5.3. $G$ is infinitesimal if and only if $G_{\varepsilon}=0$.

Proof. If $G$ is infinitesimal then $R_{0}(G)=L_{0}(G)=0$, so $0 \leq G_{\varepsilon} \leq 0$ by Lemma 5.2. Conversely, suppose $G_{\varepsilon}=0$. Then by Lemma 5.1,

$$
R_{0}(G)=R_{0}\left(G_{\varepsilon}\right)=0=L_{0}\left(G_{\varepsilon}\right)=L_{0}(G),
$$

so $G$ is infinitesimal. 
THEOREM 5.4. Using the representation $-G=\left\{-G^{R} \mid-G^{L}\right\}$, we have

$$
(-G)_{\varepsilon}=-\left(G_{\varepsilon}\right) .
$$

PROOF. Trivial if $G$ is a number; otherwise, by induction:

$$
\begin{aligned}
(-G)_{\varepsilon} & =\left\{\left(-G^{R}\right)_{\varepsilon}-\varepsilon \mid\left(-G^{L}\right)_{\varepsilon}+\varepsilon\right\} \\
& =\left\{-\left(G_{\varepsilon}^{R}\right)-\varepsilon \mid-\left(G_{\varepsilon}^{L}\right)+\varepsilon\right\} \\
& =-\left\{G_{\varepsilon}^{L}-\varepsilon \mid G_{\varepsilon}^{R}+\varepsilon\right\} \\
& =-\left(G_{\varepsilon}\right) .
\end{aligned}
$$

LEMMA 5.5. Let $G, H$ be any games.

(a) If $G+H \geq 0$, then $G_{\varepsilon}+H_{\varepsilon} \geq 0$.

(b) If $G+H \bowtie 0$, then $G_{\varepsilon}+H_{\varepsilon}+\varepsilon \bowtie 0$.

Proof. If $G$ and $H$ are numbers, (a) and (b) are immediate. Otherwise, we may assume that $G$ is not a number, and we proceed by simultaneous induction on (a) and (b).

(a) Suppose that $G+H \geq 0$ and that Right moves in $G_{\varepsilon}+H_{\varepsilon}$ to $G_{\varepsilon}^{R}+H_{\varepsilon}+\varepsilon$ (we can assume that Right's move is in $G_{\varepsilon}$ by symmetry and the Number Avoidance Theorem). Since $G^{R}+H \bowtie 0$, by induction $G_{\varepsilon}^{R}+H_{\varepsilon}+\varepsilon \triangleright 0$, so Left wins; hence $G_{\varepsilon}+H_{\varepsilon} \geq 0$.

(b) If $G+H \bowtie 0$ then Left has a winning move, say to $G^{L}+H \geq 0$ (again, by symmetry and the Number Avoidance Theorem we can assume that Left's winning move is in $G$ ). Then $G_{\varepsilon}^{L}+H_{\varepsilon} \geq 0$ by induction, so Left also has a winning move from $G_{\varepsilon}+H_{\varepsilon}+\varepsilon$.

COROLLARY 5.6. If $G \geq H$, then $G_{\varepsilon} \geq H_{\varepsilon}$.

PROOF. Using Theorem 5.4 and Lemma 5.5,

$$
G+(-H) \geq 0 \Rightarrow G_{\varepsilon}+(-H)_{\varepsilon} \geq 0 \Rightarrow G_{\varepsilon} \geq H_{\varepsilon} .
$$

THEOREM 5.7. If $G=H$, then $G_{\varepsilon}=H_{\varepsilon}$.

Proof. Follows immediately from Corollary 5.6 since $G \geq H$ and $H \geq G$.

By Theorem 5.7, $G_{\varepsilon}$ does not depend on the formal representation of $G$. Therefore, the mapping $G \mapsto G_{\varepsilon}$ is well-defined. We next show that it is a homomorphism.

LEMMA 5.8. If $x$ is a number, then $(G+x)_{\varepsilon}=G_{\varepsilon}+x$. 
Proof. The conclusion is trivial if $G$ is a number. Otherwise, $G+x$ is not a number, so by induction and the Number Translation Theorem

$$
\begin{aligned}
(G+x)_{\varepsilon} & =\left\{G^{L}+x \mid G^{R}+x\right\}_{\varepsilon} \\
& =\left\{\left(G^{L}+x\right)_{\varepsilon}-\varepsilon \mid\left(G^{R}+x\right)_{\varepsilon}+\varepsilon\right\} \\
& =\left\{G_{\varepsilon}^{L}-\varepsilon+x \mid G_{\varepsilon}^{R}+\varepsilon+x\right\}
\end{aligned}
$$

If $G_{\varepsilon}$ is not a number, then this is equal to $G_{\varepsilon}+x$ by the Number Translation Theorem. Otherwise, there is some number $z$ such that

$$
G_{\varepsilon}^{L}-\varepsilon \triangleleft \mid z \triangleleft G_{\varepsilon}^{R}+\varepsilon .
$$

Translating by $x$, we have that

$$
G_{\varepsilon}^{L}-\varepsilon+x \triangleleft|z+x \triangleleft| G_{\varepsilon}^{R}+\varepsilon+x,
$$

and since $(G+x)_{\varepsilon}=\left\{G_{\varepsilon}^{L}-\varepsilon+x \mid G_{\varepsilon}^{R}+\varepsilon+x\right\}$, it follows that $(G+x)_{\varepsilon}$ is a number. Then by Lemma 5.1, we conclude that

$(G+x)_{\varepsilon}=L_{0}\left((G+x)_{\varepsilon}\right)=L_{0}(G+x)=L_{0}(G)+x=L_{0}\left(G_{\varepsilon}\right)+x=G_{\varepsilon}+x$.

The following theorem establishes that reduction by $\varepsilon$ is a projection: an idempotent homomorphism.

THEOREM 5.9. Let $G, H$ be any games. Then:

(a) $(G+H)_{\varepsilon}=G_{\varepsilon}+H_{\varepsilon}$.

(b) $G_{\varepsilon} \equiv_{\text {Inf }} G$.

(c) $\left(G_{\varepsilon}\right)_{\varepsilon}=G_{\varepsilon}$.

PROOF. (a) This reduces to Lemma 5.8 if either $G$ or $H$ is a number. Otherwise, there are two cases. First, if $x=G+H$ is a number, then by Theorem 5.4 and Lemma 5.8 we have

$$
(G+H)_{\varepsilon}-G_{\varepsilon}=x-G_{\varepsilon}=(x-G)_{\varepsilon}=H_{\varepsilon} .
$$

Finally, if none of $G, H, G+H$ are numbers, then by induction

$$
\begin{aligned}
(G+H)_{\varepsilon} & =\left\{\left(G^{L}+H\right)_{\varepsilon}-\varepsilon,\left(G+H^{L}\right)_{\varepsilon}-\varepsilon \mid\left(G^{R}+H\right)_{\varepsilon}+\varepsilon,\left(G+H^{R}\right)_{\varepsilon}+\varepsilon\right\} \\
& =\left\{\left(G_{\varepsilon}^{L}-\varepsilon\right)+H_{\varepsilon}, G_{\varepsilon}+\left(H_{\varepsilon}^{L}-\varepsilon\right) \mid\left(G_{\varepsilon}^{R}+\varepsilon\right)+H_{\varepsilon}, G_{\varepsilon}+\left(H^{R}+\varepsilon\right)\right\} \\
& =G_{\varepsilon}+H_{\varepsilon} .
\end{aligned}
$$

(b) follows by induction and Theorem 2.10 .

(c) Using (a), (b), and Theorem 5.3, we obtain

$$
0=\left(G_{\varepsilon}-G\right)_{\varepsilon}=\left(G_{\varepsilon}\right)_{\varepsilon}-G_{\varepsilon}
$$


THEOREM 5.10. The mapping $G \mapsto G_{\varepsilon}$ is a reduction.

ProOF. $G \equiv_{\operatorname{Inf}} G_{\varepsilon}$ by Theorem 5.9, and $x_{\varepsilon}=x$ for numbers $x$ by definition. Finally, if $G \equiv_{\operatorname{Inf}} H$, then $G=H+\delta$ for some infinitesimal $\delta$, so by Theorems 5.3 and $5.9, G_{\varepsilon}=H_{\varepsilon}+\delta_{\varepsilon}=H_{\varepsilon}$.

We conclude with a theorem on incentives for games reduced by $\varepsilon$ whose elegant proof makes it deserving of presentation.

THEOREM 5.11. If $G_{\varepsilon}$ is not a number, then $G_{\varepsilon}$ has at least one Left incentive and at least one Right incentive that are $\geq-\varepsilon$.

Proof. By Theorem 2.9, there is some Left option $G_{\varepsilon}^{L}-\varepsilon$ of $G_{\varepsilon}$ and some infinitesimal $\delta$ such that

$$
\left(G_{\varepsilon}^{L}-\varepsilon\right)-G_{\varepsilon} \geq \delta .
$$

Reducing by $\varepsilon$ gives $\left(G_{\varepsilon}^{L}\right)_{\varepsilon}-\varepsilon_{\varepsilon}-\left(G_{\varepsilon}\right)_{\varepsilon} \geq \delta_{\varepsilon}$ by Theorem 5.9(a) and Corollary 5.6, which implies $G_{\varepsilon}^{L}-G_{\varepsilon} \geq 0$ by Theorems 5.3 and 5.9(c), and finally

$$
\left(G_{\varepsilon}^{L}-\varepsilon\right)-G_{\varepsilon} \geq-\varepsilon
$$

The proof for Right incentives is identical.

\section{All-small reductions}

Our final task is to relate the two reductions presented thus far. Recall that a game is all-small if all of its followers are infinitesimal. We will see that when $\varepsilon$ is all-small, $G_{\varepsilon}$ can be computed by adding appropriate multiples of $\varepsilon$ to the stops of $\bar{G}$. We begin with a simple observation:

LEMMA 6.1. If $G_{\varepsilon}$ is not a number, then there is some $G_{\varepsilon}^{L}$ with $R_{0}\left(G_{\varepsilon}^{L}\right)>$ $R_{0}\left(G_{\varepsilon}\right)$.

Proof. Since $G_{\varepsilon}$ is not a number, $G$ is not numberish. Hence $L_{0}(G)>R_{0}(G)$, so we can choose any $G_{\varepsilon}^{L}$ where $R_{0}\left(G^{L}\right)=L_{0}(G)$.

The following theorem strengthens Theorem 2.9 when $\varepsilon$ is all-small.

Theorem 6.2 (All-Small Avoidance Theorem). If $\varepsilon \mathbb{\triangleright} \triangleright 0$ is all-small and $G_{\varepsilon}$ is not a number, then $G_{\varepsilon}$ has at least one Left incentive and at least one Right incentive that exceed every incentive (Left and Right) of $\varepsilon$.

The hypothesis that $\varepsilon$ is all-small is essential. For example, if we take $G= \pm 1$ and $\varepsilon=2$, then $G_{\varepsilon}= \pm\{3 \mid 1 \| 1\}$. We can verify that the Left incentive of $G_{\varepsilon}$ is less than the Right incentive of 2.

Proof of Theorem 6.2. We will show that $G_{\varepsilon}$ has a Left incentive that exceeds every incentive of $\varepsilon$; the proof for Right incentives is identical. First, for 
any game $H$, we define the Left and Right parities $p^{L}(H), p^{R}(H)$ as follows. If $H$ is numberish, then $p^{L}(H)=p^{R}(H)=0$. Otherwise,

$$
p^{L}(H)= \begin{cases}1 & \text { if } p^{R}\left(H^{L}\right)=0 \text { for every } H^{L} \text { with } R_{0}\left(H^{L}\right)=L_{0}(H), \\ 0 & \text { if } p^{R}\left(H^{L}\right)=1 \text { for any such } H^{L} .\end{cases}
$$

$p^{R}(H)$ is defined similarly, with $L$ and $R$ interchanged.

Since $G_{\varepsilon}$ is not a number, neither is $G$, so there is some $G^{L}$ with $R_{0}\left(G^{L}\right)=$ $L_{0}(G)$. If $p^{L}(G)=0$ then we can choose such $G^{L}$ with $p^{R}\left(G^{L}\right)=1$, so we can guarantee that at least one of $p^{R}\left(G^{L}\right), p^{L}(G)$ is 1 . We claim that for each (Left or Right) incentive $\Delta$ of $\varepsilon$,

$$
\left(G_{\varepsilon}^{L}-\varepsilon\right)-G_{\varepsilon} \geq \Delta .
$$

Observe that $p^{R}(-H)=p^{L}(H)$ and $p^{L}(-H)=p^{R}(H)$ (proof by simple induction). It therefore suffices to prove the following: if Right moves from any position of the form

$$
A_{\varepsilon}+B_{\varepsilon}-\varepsilon-\Delta, \quad \text { with } R_{0}(A)+R_{0}(B)=0, p^{R}(A)+p^{R}(B) \geq 1,
$$

then Left can either win outright, or respond to another position of the same form. The following proof makes heavy use of the inequality $R_{0}(A+B) \geq$ $R_{0}(A)+R_{0}(B)$ from Proposition 2.5(b).

Since $p^{R}(A)+p^{R}(B) \geq 1, A_{\varepsilon}$ and $B_{\varepsilon}$ cannot both be numbers. It follows that Right can never move in $(-\varepsilon-\Delta)$, since by Lemma 6.1 this would allow Left to move in one of $A_{\varepsilon}$ or $B_{\varepsilon}$ leaving a position $H$ with $R_{0}(H)>0$ (here we use the fact that $\varepsilon$ is all-small which implies that none of its incentives or followers contribute to the stops of the entire game).

Next suppose that Right moves in $A_{\varepsilon}$ from (6-1). By the Number Avoidance Theorem, we may assume that $A_{\varepsilon}$ is not a number. There are three cases.

Case 1: $A_{\varepsilon}^{R}$ is not a number. Then Left moves to $A_{\varepsilon}^{R L}$ with $R_{0}\left(A^{R L}\right)=$ $L_{0}\left(A^{R}\right) \geq R_{0}(A)$. In this case the position is

$$
H=A_{\varepsilon}^{R L}+B_{\varepsilon}-\varepsilon-\Delta, \quad \text { with } R_{0}\left(A^{R L}\right)+R_{0}(B) \geq 0 .
$$

If the inequality is strict, then since $-\varepsilon-\Delta$ is infinitesimal, $R_{0}(H)>0$ and Left wins outright. Otherwise, either $p^{R}(B)=1$ or $p^{R}(A)=1$; in the latter case $p^{L}\left(A^{R}\right)=0$, so Left can choose $A^{R L}$ with $p^{R}\left(A^{R L}\right)=p^{R}(A)=1$. In both cases, Left leaves a smaller position of the form (6-1).

Case 2: $A_{\varepsilon}^{R}$ is a number, but $B_{\varepsilon}$ is not. Then $R_{0}\left(A^{R}\right)=L_{0}\left(A^{R}\right) \geq R_{0}(A)$, so by Lemma 6.1 Left can move in $B_{\varepsilon}$ leaving a position $H$ with $R_{0}(H)>0$, and Left wins outright. 
Case 3: Both $A_{\varepsilon}^{R}$ and $B_{\varepsilon}$ are numbers. Then $A_{\varepsilon}^{R}=L_{0}\left(A^{R}\right) \geq R_{0}(A)=$ $-R_{0}(B)=-B_{\varepsilon}$. If the inequality is strict, then Left wins outright. Otherwise, the overall position is

$$
\left(A_{\varepsilon}^{R}+\varepsilon\right)+B_{\varepsilon}-\varepsilon-\Delta=-\Delta .
$$

But $\Delta$ is an incentive, so necessarily $\Delta \triangleleft \mid 0$, whence $-\Delta \mid \triangleright 0$.

This exhausts Right's moves in $A_{\varepsilon}$ from (6-1). The situation is identical if Right moves in $B_{\varepsilon}$, so the proof is complete.

Corollary 6.3 (All-SMAll TRANSLATION THEOREM). If $G$ is not a number, $n$ is an integer and $\varepsilon \bowtie 0$ is all-small, then $G_{\varepsilon}+n \cdot \varepsilon=\left\{G_{\varepsilon}^{L}+(n-1) \cdot \varepsilon \mid\right.$ $\left.G_{\varepsilon}^{R}+(n+1) \cdot \varepsilon\right\}$.

PROOF. This is trivial if $n=0$. Otherwise the game is either $G_{\varepsilon}+\varepsilon+\varepsilon+\cdots$ or $G_{\varepsilon}-\varepsilon-\varepsilon-\cdots ;$ in either case the Left and Right incentives of $\pm \varepsilon$ are dominated by incentives of $G_{\varepsilon}$ by Theorem 6.2.

This translation theorem allows us to quickly compute $G_{\varepsilon}$ from its definition by absorbing the $\pm \varepsilon$ terms in the followers of $G_{\varepsilon}$ until we reach the stops. It is straightforward (although the notation is cumbersome) to determine the multiple of $\varepsilon$ which must be added to each stop:

DEFinition 6.4. Let $G^{X Y Z \ldots}$ be a follower of $G$ where each of $X, Y, Z, \ldots$ denotes a Left or Right option. The weight $w_{G}\left(G^{X Y Z \cdots)}\right.$ of $G^{X Y Z \cdots}$ is the number of Left options in $X, Y, Z, \ldots$ minus the number of Right options in $X, Y, Z, \ldots$

Note that we have already encountered the concept of weight disguised as temper in Section 3; a representation of a game is even- (odd-) tempered if the stops all have even (odd) weight. As an example, in the game $\{2 \| 1 \mid 0\}$, the stop 2 has weight 1 since it is reached by a single Left move, the stop 1 has weight 0 since it is reached by a Right move followed by a Left move, and the stop 0 has weight -2 since it is reached by two Right moves.

THEOREM 6.5. If $\varepsilon \bowtie 0$ is all-small, then $G_{\varepsilon}$ is the game obtained from $\bar{G}$ by replacing each stop $H$ with $H-w_{\bar{G}}(H) \cdot \varepsilon$.

Proof. Since $\bar{G} \equiv_{\text {Inf }} G$, we have $G_{\varepsilon}=(\bar{G})_{\varepsilon}$. With this observation, the theorem follows immediately from Corollary 6.3 and the definition of reduction by $\varepsilon$ applied to $\bar{G}$.

For example, if $G=\{2 \| 1 \mid 0\}$ then $G_{\uparrow}=\{2 \downarrow \| 1 \mid \Uparrow\}$. Note that for $\varepsilon=*$, Theorem 6.5 agrees with our results from Sections 3 and 4 as it states that $G_{*}$ is obtained from $\bar{G}$ by adding $*$ to the stops having odd weight. 


\section{Conclusion and open problems}

The reduced canonical form is a valuable tool in the study of combinatorial games; see [4, Section 7] in this volume for an example of its successful application. However, there are several potentially useful directions in which these ideas can be extended.

Section 5 does not completely characterize the reductions that are also homomorphisms. For example, the reader might wish to verify that the mapping $\rho$ given by

$\rho(G)= \begin{cases}G & \text { if } G \text { is a number, } \\ \left\{\rho\left(G^{L}\right)-*, \rho\left(G^{L}\right)-* 2 \mid \rho\left(G^{R}\right)+*, \rho\left(G^{R}\right)+* 2\right\} & \text { otherwise }\end{cases}$

is both a reduction and a homomorphism. In fact, we could replace $*$ and $* 2$ by any finite set of infinitesimals $\triangleright \triangleright 0$ : the results of Section 5 all apply with virtually unchanged proofs. It would be interesting to investigate other reduction-homomorphisms (if indeed they exist).

Open PRoblem. Give a complete characterization of all reduction-homomorphisms $\rho: \mathcal{G} \rightarrow \mathcal{G}$.

Another important question is: to what extent can these constructions be generalized to groups other than Inf? In particular, if $\mathcal{K}$ is any subgroup of $\mathcal{G}$, then we can define a reduction modulo $\mathcal{K}$ as a map that isolates a unique element of each $\mathcal{K}$-equivalence class.

As a typical example, consider the group of infinitesimals of order $n$ :

$$
\operatorname{Inf}^{n}=\left\{G: k \cdot \downarrow_{n}<G<k \cdot \uparrow^{n} \text { for some } k\right\} .
$$

OPEN PROBLEM. Give an effective construction for reduction modulo $\mathrm{Inf}^{n}$ (or some other useful class of games).

There are many interesting games in which all positions are infinitesimals, and reduction modulo Inf is obviously unhelpful in studying such games. The theory of atomic weights is sometimes useful, but quite often one encounters large classes of positions with atomic weight zero. In such cases, reduction modulo Inf $^{2}$ could be a productive tool.

Generalizing beyond short games, reduced canonical forms can be suitably defined for a certain class of well-behaved loopy games known as stoppers (see [1] or [6] for a definition). That construction is beyond the scope of this paper, but see [5, Section 5.4] for a complete discussion.

\section{References}

[1] E. R. Berlekamp, J. H. Conway, and R. K. Guy. Winning Ways for Your Mathematical Plays. A. K. Peters, Ltd., Natick, MA, second edition, 2001. 
[2] D. Calistrate. The reduced canonical form of a game. In R. J. Nowakowski, editor, Games of No Chance, number 29 in MSRI Publications, pages 409-416. Cambridge University Press, Cambridge, 1996.

[3] J. H. Conway. On Numbers and Games. A. K. Peters, Ltd., Natick, MA, second edition, 2001.

[4] G. A. Mesdal. Partizan Splittles. In this volume. 2006.

[5] A. N. Siegel. Loopy Games and Computation. PhD thesis, University of California at Berkeley, 2005.

[6] A. N. Siegel. Coping with cycles. In this volume. 2006.

J.P. GROSSMAN

D. E. Shaw RESEARCH

jpg@alum.mit.edu

Aaron N. Siegel

aaron.n.siegel@gmail.com 
